\title{
Hermite-Hadamard-Fejer type inequalities for GA- $s$ convex functions via fractional integrals
}

\author{
Imdat Iscan ${ }^{1}$ and Mehmet Kunt ${ }^{2}$ \\ ${ }^{1}$ Department of Mathematics, Faculty of Sciences and Arts, Giresun University, Giresun, Turkey \\ ${ }^{2}$ Department of Mathematics, Faculty of Sciences, Karadeniz Technical University, Trabzon, Turkey
}

Received: 4 January 2016, Revised: 4 January 2016, Accepted: 1 May 2016

Published online: 26 May 2016.

\begin{abstract}
In this paper, some Hermite-Hadamard-Fejer type integral inequalities for GA-s convex functions in fractional integral forms are obtained.
\end{abstract}

Keywords: Hermite-Hadamard inequality, Hermite-Hadamard-Fejer inequality, Hadamard fractional integrals, GA-s convex functions.

\section{Introduction}

Let $f: I \subset \mathbb{R} \rightarrow \mathbb{R}$ be a convex function defined on the interval $I$ of real numbers and $a, b \in I$ with $a<b$. The inequality

$$
f\left(\frac{a+b}{2}\right) \leq \frac{1}{b-a} \int_{a}^{b} f(x) d x \leq \frac{f(a)+f(b)}{2}
$$

is well known in the literature as Hermite-Hadamard's inequality [2].

The most well-known inequalities related to the integral mean of a convex function $f$ are the Hermite Hadamard inequalities or their weighted versions, the so-called Hermite-Hadamard-Fejér inequalities.

In [1], Fejér established the following Fejér inequality which is the weighted generalization of Hermite-Hadamard inequality (1):

Theorem 1. Let $f:[a, b] \rightarrow \mathbb{R}$ be convex function. Then the inequality

$$
f\left(\frac{a+b}{2}\right) \int_{a}^{b} g(x) d x \leq \int_{a}^{b} f(x) g(x) d x \leq \frac{f(a)+f(b)}{2} \int_{a}^{b} g(x) d x
$$

holds, where $g:[a, b] \rightarrow \mathbb{R}$ is nonnegative, integrable and symmetric to $(a+b) / 2$.

For some results which generalize, improve, and extend the inequalities (1) and (2) see [3,14,15, 17].

Definition 1. [11,12]. A function $f: I \subset(0, \infty) \rightarrow \mathbb{R}$ is said to be GA-convex (geometric-arithmetically convex) if

$$
f\left(x^{t} y^{1-t}\right) \leq t f(x)+(1-t) f(y)
$$


for all $x, y \in I$ and $t \in[0,1]$.

Definition 2. [16]. Let $f: I \subset[0, \infty) \rightarrow[0, \infty)$ and $s \in(0,1]$. A function $f(x)$ is said to be GA-s convex (geomatricarithmetically s-convex) on I if

$$
f\left(x^{t} y^{1-t}\right) \leq t^{s} f(x)+(1-t)^{s} f(y)
$$

for all $x, y \in I$ and $t \in[0,1]$.

In [10], Latif et al. established the following inequality which is the weighted generalization of Hermite-Hadamard inequality for GA-convex functions as follows:

Theorem 2. Let $f: I \subset(0, \infty) \rightarrow \mathbb{R}$ be a GA-convex function and $a, b \in I$ with $a<b$. Let $g:[a, b] \rightarrow[0, \infty)$ be continuous positive mapping and geometrically symmetric to $\sqrt{a b}$. Then

$$
f(\sqrt{a b}) \int_{a}^{b} \frac{g(x)}{x} d x \leq \int_{a}^{b} \frac{f(x) g(x)}{x} d x \leq \frac{f(a)+f(b)}{2} \int_{a}^{b} \frac{g(x)}{x} d x .
$$

The following definitions and mathematical preliminaries of fractional calculus theory are used further in this paper.

Definition 3. [9]. Let $f \in L[a, b]$. The Hadamard integrals $J_{a+}^{\alpha} f$ and $J_{b-}^{\alpha} f$ of order $\alpha>0$ with $b>a \geq 0$ are defined by

$$
J_{a+}^{\alpha} f(x)=\frac{1}{\Gamma(\alpha)} \int_{a}^{x}\left(\ln \frac{x}{t}\right)^{\alpha-1} f(t) \frac{d t}{t}, x>a
$$

and

$$
J_{b-}^{\alpha} f(x)=\frac{1}{\Gamma(\alpha)} \int_{x}^{b}\left(\ln \frac{t}{x}\right)^{\alpha-1} f(t) \frac{d t}{t}, x<b
$$

respectively, where $\Gamma(\alpha)$ is the Gamma function defined by $\Gamma(\alpha)=\int_{0}^{\infty} e^{-t} t^{\alpha-1} d t$.

Because of the wide application of Hermite-Hadamard type inequalities and fractional integrals, many researchers extend their studies to Hermite-Hadamard type inequalities involving fractional integrals not limited to integer integrals. Recently, more and more Hermite-Hadamard inequalities involving fractional integrals have been obtained for different classes of functions; see $[4,5,6,7,18,19]$.

In [5], Iscan presented Hermite-Hadamard's inequalities for GA-convex functions in fractional integral forms as follows:

Theorem 3. Let $f: I \subset(0, \infty) \rightarrow \mathbb{R}$ be a function such that $f \in L[a, b]$ where $a, b \in I$ with $a<b$. If $f$ is a GA-convex function on $[a, b]$, then the following inequalities for fractional integrals holds:

$$
f(\sqrt{a b}) \leq \frac{\Gamma(\alpha+1)}{2\left(\ln \frac{b}{a}\right)^{\alpha}}\left[J_{a+}^{\alpha} f(b)+J_{b-}^{\alpha} f(a)\right] \leq \frac{f(a)+f(b)}{2}
$$

with $\alpha>0$.

In [8], the authors presented Hermite-Hadamard-Fejer inequalities for GA-convex functions in fractional integral forms as follows:

Theorem 4. Let $f:[a, b] \subset(0, \infty) \rightarrow \mathbb{R}$ be a GA-convex function with $a<b$ and $f \in L[a, b]$. If $g:[a, b] \rightarrow \mathbb{R}$ is nonnegative, integrable and geometrically symmetric with respect to $\sqrt{a b}$, then the following inequalities for fractional integrals holds:

$$
f(\sqrt{a b})\left[J_{a+}^{\alpha} g(b)+J_{b-}^{\alpha} g(a)\right] \leq\left[J_{a+}^{\alpha}(f g)(b)+J_{b-}^{\alpha}(f g)(a)\right] \leq \frac{f(a)+f(b)}{2}\left[J_{a+}^{\alpha} g(b)+J_{b-}^{\alpha} g(a)\right]
$$


with $\alpha>0$.

Lemma 1. [8]. Let $f:[a, b] \subset(0, \infty) \rightarrow \mathbb{R}$ be a differentiable mapping on $(a, b)$ with $a<b$ and $f^{\prime} \in L[a, b]$. If $g:[a, b] \rightarrow \mathbb{R}$ is integrable and geometrically symmetric with respect to $\sqrt{a b}$ then the following equality for fractional integrals holds:

$$
\left(\frac{f(a)+f(b)}{2}\right)\left[J_{a+}^{\alpha} g(b)+J_{b--}^{\alpha} g(a)\right]-\left[J_{a+}^{\alpha}(f g)(b)+J_{b-}^{\alpha}(f g)(a)\right]=\frac{1}{\Gamma(\alpha)} \int_{a}^{b}\left[\begin{array}{c}
\int_{a}^{t}\left(\ln \frac{b}{s}\right)^{\alpha-1} g(s) \frac{d s}{s} \\
-\int_{t}^{b}\left(\ln \frac{s}{a}\right)^{\alpha-1} g(s) \frac{d s}{s}
\end{array}\right] f^{\prime}(t) d t
$$

with $\alpha>0$.

Lemma 2. [13,19]. For $0<\alpha \leq 1$ and $0 \leq a<b$, we have

$$
\left|a^{\alpha}-b^{\alpha}\right| \leq(b-a)^{\alpha}
$$

In this paper, we obtain some new inequalities connected with the right-hand side of Hermite-Hadamard-Fejér type integral inequality for GA-s convex function in fractional integral forms.

\section{Main results}

Throughout this section, let $\|g\|_{\infty}=\sup _{t \in[a, b]}|g(t)|$, for the continuous function $g:[a, b] \rightarrow \mathbb{R}$.

Theorem 5. Let $f: I \subset(0, \infty) \rightarrow \mathbb{R}$ be a differentiable mapping on $I^{\circ}$ where $a, b \in I$ with $a<b$ and $f^{\prime} \in L[a, b]$. If $\left|f^{\prime}\right|$ is GA-s convex on $[a, b], g:[a, b] \rightarrow \mathbb{R}$ is continuous and geometrically symmetric with respect to $\sqrt{a b}$, then the following inequality for fractional integrals holds:

$$
\left|\begin{array}{c}
\left(\frac{f(a)+f(b)}{2}\right)\left[J_{a+}^{\alpha} g(b)+J_{b-}^{\alpha} g(a)\right] \\
-\left[J_{a+}^{\alpha}(f g)(b)+J_{b-}^{\alpha}(f g)(a)\right]
\end{array}\right| \leq \frac{\|g\|_{\infty} \ln ^{\alpha+1}\left(\frac{b}{a}\right)}{\Gamma(\alpha+1)}\left[C_{1}(\alpha)\left|f^{\prime}(a)\right|+C_{2}(\alpha)\left|f^{\prime}(b)\right|\right],
$$

where

$$
\begin{gathered}
C_{1}(\alpha)=\left[\begin{array}{c}
\int_{0}^{\frac{1}{2}}\left[(1-u)^{\alpha}-u^{\alpha}\right](1-u)^{s} a^{1-u} b^{u} d u \\
+\int_{\frac{1}{2}}^{1}\left[u^{\alpha}-(1-u)^{\alpha}\right](1-u)^{s} a^{1-u} b^{u} d u
\end{array}\right], \\
C_{2}(\alpha)=\left[\begin{array}{c}
\int_{0}^{\frac{1}{2}}\left[(1-u)^{\alpha}-u^{\alpha}\right] u^{s} a^{1-u} b^{u} d u \\
+\int_{\frac{1}{2}}^{1}\left[u^{\alpha}-(1-u)^{\alpha}\right] u^{s} a^{1-u} b^{u} d u
\end{array}\right],
\end{gathered}
$$

with $\alpha>0$.

Proof. From Lemma 1 we have

$$
\left|\begin{array}{c}
\left(\frac{f(a)+f(b)}{2}\right)\left[J_{a+}^{\alpha} g(b)+J_{b-}^{\alpha} g(a)\right] \\
-\left[J_{a+}^{\alpha}(f g)(b)+J_{b-}^{\alpha}(f g)(a)\right]
\end{array}\right| \leq \frac{1}{\Gamma(\alpha)} \int_{a}^{b}\left|\int_{a}^{t}\left(\ln \frac{b}{s}\right)^{\alpha-1} g(s) \frac{d s}{s}-\int_{t}^{b}\left(\ln \frac{s}{a}\right)^{\alpha-1} g(s) \frac{d s}{s}\right|\left|f^{\prime}(t)\right| d t .
$$

Setting $t=a^{1-u} b^{u}$ and $d t=a^{1-u} b^{u} \ln \left(\frac{b}{a}\right) d u$ gives

$$
\left|\begin{array}{c}
\left(\frac{f(a)+f(b)}{2}\right)\left[J_{a+}^{\alpha} g(b)+J_{b-}^{\alpha} g(a)\right] \\
-\left[J_{a+}^{\alpha}(f g)(b)+J_{b-}^{\alpha}(f g)(a)\right]
\end{array}\right| \leq \frac{1}{\Gamma(\alpha)} \int_{0}^{1}\left|\begin{array}{c}
\int_{a}^{a^{1-u} b^{u}}\left(\ln \frac{b}{s}\right)^{\alpha-1} g(s) \frac{d s}{s} \\
-\int_{a^{1-u} b^{u}}^{b}\left(\ln \frac{s}{a}\right)^{\alpha-1} g(s) \frac{d s}{s}
\end{array}\right|\left|f^{\prime}\left(a^{1-u} b^{u}\right)\right| a^{1-u} b^{u} \ln \left(\frac{b}{a}\right) d u .
$$


Since $g:[a, b] \rightarrow \mathbb{R}$ is geometrically symmetric with respect to $\sqrt{a b}$ we write

$$
\int_{a^{1-u} b^{u}}^{b}\left(\ln \frac{s}{a}\right)^{\alpha-1} g(s) \frac{d s}{s}=\int_{a}^{a^{u} b^{1-u}}\left(\ln \frac{b}{s}\right)^{\alpha-1} g\left(\frac{a b}{s}\right) \frac{d s}{s}=\int_{a}^{a^{u} b^{1-u}}\left(\ln \frac{b}{s}\right)^{\alpha-1} g(s) \frac{d s}{s} .
$$

Then we have

$$
\begin{aligned}
& \left|\int_{a}^{a^{1-u} b^{u}}\left(\ln \frac{b}{s}\right)^{\alpha-1} g(s) \frac{d s}{s}-\int_{a^{1-u} b^{u}}^{b}\left(\ln \frac{s}{a}\right)^{\alpha-1} g(s) \frac{d s}{s}\right|=\left|\int_{a^{1-u} b^{u}}^{a^{u} b^{1-u}}\left(\ln \frac{b}{s}\right)^{\alpha-1} g(s) \frac{d s}{s}\right| \\
& \leq\left\{\begin{array}{l}
\int_{a^{u} b^{1-u} u^{1-u}}^{a^{u}}\left(\ln \frac{b}{s}\right)^{\alpha-1}|g(s)| \frac{d s}{s} u \in\left[0, \frac{1}{2}\right] \\
\int_{a^{u} b^{1-u}}^{a^{1-u} b^{u}}\left(\ln \frac{b}{s}\right)^{\alpha-1}|g(s)| \frac{d s}{s} u \in\left[\frac{1}{2}, 1\right]
\end{array}\right. \\
& \leq\|g\|_{\infty}\left\{\begin{array}{l}
\int_{a^{1}-u}^{a^{u} b^{1-u}}\left(\ln \frac{b}{s}\right)^{\alpha-1} \frac{d s}{s} u \in\left[0, \frac{1}{2}\right] \\
\int_{a^{u} b^{1}-u}^{a^{1-u} b^{u}}\left(\ln \frac{b}{s}\right)^{\alpha-1} \frac{d s}{s} u \in\left[\frac{1}{2}, 1\right]
\end{array}\right. \\
& =\|g\|_{\infty} \frac{\left(\ln \frac{b}{a}\right)^{\alpha}}{\alpha}\left\{\begin{array}{l}
(1-u)^{\alpha}-u^{\alpha} u \in\left[0, \frac{1}{2}\right] \\
u^{\alpha}-(1-u)^{\alpha} u \in\left[\frac{1}{2}, 1\right]
\end{array}\right. \text {. }
\end{aligned}
$$

Since $\left|f^{\prime}\right|$ is GA-s convex on $[a, b]$, we have

$$
\left|f^{\prime}\left(a^{1-u} b^{u}\right)\right| \leq(1-u)^{s}\left|f^{\prime}(a)\right|+u^{s}\left|f^{\prime}(b)\right|
$$

A combination (8), (9) and (10)

$$
\begin{aligned}
& \left|\begin{array}{c}
\left(\frac{f(a)+f(b)}{2}\right)\left[J_{a+g}^{\alpha} g(b)+J_{b-}^{\alpha} g(a)\right] \\
-\left[J_{a+}^{\alpha}(f g)(b)+J_{b-}^{\alpha}(f g)(a)\right]
\end{array}\right| \leq \frac{1}{\Gamma(\alpha)} \int_{0}^{1}\left|\begin{array}{c}
\int_{a}^{a^{1-u} b^{u}}\left(\ln \frac{b}{s}\right)^{\alpha-1} g(s) \frac{d s}{s} \\
-\int_{a^{1-u} b^{u}}^{b}\left(\ln \frac{s}{a}\right)^{\alpha-1} g(s) \frac{d s}{s}
\end{array}\right|\left|f^{\prime}\left(a^{1-u} b^{u}\right)\right| a^{1-u} b^{u} \ln \left(\frac{b}{a}\right) d u . \\
& \leq \frac{1}{\Gamma(\alpha)} \int_{0}^{\frac{1}{2}}\left(\|g\|_{\infty} \frac{\left(\ln \frac{b}{a}\right)^{\alpha}}{\alpha}\left[(1-u)^{\alpha}-u^{\alpha}\right]\right)\left[\begin{array}{c}
(1-u)^{s}\left|f^{\prime}(a)\right| \\
+u^{s}\left|f^{\prime}(b)\right|
\end{array}\right] a^{1-u} b^{u} \ln \left(\frac{b}{a}\right) d u \\
& +\frac{1}{\Gamma(\alpha)} \int_{\frac{1}{2}}^{1}\left(\|g\|_{\infty} \frac{\left(\ln \frac{b}{a}\right)^{\alpha}}{\alpha}\left[u^{\alpha}-(1-u)^{\alpha}\right]\right)\left[\begin{array}{c}
(1-u)^{s}\left|f^{\prime}(a)\right| \\
+u^{s}\left|f^{\prime}(b)\right|
\end{array}\right] a^{1-u} b^{u} \ln \left(\frac{b}{a}\right) d u \\
& \leq \frac{\ln ^{\alpha+1}\left(\frac{b}{a}\right)\|g\|_{\infty}}{\Gamma(\alpha+1)}\left\{\int_{0}^{\frac{1}{2}}\left[(1-u)^{\alpha}-u^{\alpha}\right]\left[(1-u)^{s}\left|f^{\prime}(a)\right|+u^{s}\left|f^{\prime}(b)\right|\right] a^{1-u} b^{u} d u\right. \\
& \left.+\int_{\frac{1}{2}}^{1}\left[u^{\alpha}-(1-u)^{\alpha}\right]\left[(1-u)^{s}\left|f^{\prime}(a)\right|+u^{s}\left|f^{\prime}(b)\right|\right] a^{1-u} b^{u} d u\right\} \\
& =\frac{\ln ^{\alpha+1}\left(\frac{b}{a}\right)\|g\|_{\infty}}{\Gamma(\alpha+1)} \\
& \times\left\{\left[\begin{array}{c}
\int_{0}^{\frac{1}{2}}\left[(1-u)^{\alpha}-u^{\alpha}\right](1-u)^{s} a^{1-u} b^{u} d u \\
+\int_{\frac{1}{2}}^{1}\left[u^{\alpha}-(1-u)^{\alpha}\right](1-u)^{s} a^{1-u} b^{u} d u
\end{array}\right]\left|f^{\prime}(a)\right|\right. \\
& \left.+\left[\begin{array}{c}
\int_{0}^{\frac{1}{2}}\left[(1-u)^{\alpha}-u^{\alpha}\right] u^{s} a^{1-u} b^{u} d u \\
+\int_{\frac{1}{2}}^{1}\left[u^{\alpha}-(1-u)^{\alpha}\right] u^{s} a^{1-u} b^{u} d u
\end{array}\right]\left|f^{\prime}(b)\right|\right\} \text {. }
\end{aligned}
$$

This completes the proof.

Corollary 1. In Theorem 5;

(1) If we take $\alpha=1$ we have the following Hermite-Hadamard-Fejer inequality for GA-s convex functions which is related 
to the right-hand side of (3):

$$
\left|\frac{f(a)+f(b)}{2} \int_{a}^{b} \frac{g(x)}{x} d x-\int_{a}^{b} \frac{f(x) g(x)}{x} d x\right| \leq \frac{\|g\|_{\infty} \ln ^{2}\left(\frac{b}{a}\right)}{2}\left[C_{1}(1)\left|f^{\prime}(a)\right|+C_{2}(1)\left|f^{\prime}(b)\right|\right],
$$

(2) If we take $g(x)=1$ we have the following Hermite-Hadamard inequality for GA-s convex functions in fractional integral forms which is related to the right-hand side of (4):

$$
\left|\frac{f(a)+f(b)}{2}-\frac{\Gamma(\alpha+1)}{2\left(\ln \frac{b}{a}\right)^{\alpha}}\left[J_{a+}^{\alpha} f(b)+J_{b-}^{\alpha} f(a)\right]\right| \leq \frac{\ln \left(\frac{b}{a}\right)}{2}\left[C_{1}(\alpha)\left|f^{\prime}(a)\right|+C_{2}(\alpha)\left|f^{\prime}(b)\right|\right],
$$

(3) If we take $\alpha=1$ and $g(x)=1$ we have the following Hermite-Hadamard inequality for GA-s convex functions:

$$
\left|\frac{f(a)+f(b)}{2}-\frac{1}{\ln \frac{b}{a}} \int_{a}^{b} \frac{f(x)}{x} d x\right| \leq \frac{\ln \left(\frac{b}{a}\right)}{2}\left[C_{1}(1)\left|f^{\prime}(a)\right|+C_{2}(1)\left|f^{\prime}(b)\right|\right] .
$$

Theorem 6. Let $f: I \subset(0, \infty) \rightarrow \mathbb{R}$ be a differentiable mapping on $I^{\circ}$ where $a, b \in I$ with $a<b$ and $f^{\prime} \in L[a, b]$. If $\left|f^{\prime}\right|^{q}$, $q \geq 1$ is GA-s convex on $[a, b], g:[a, b] \rightarrow \mathbb{R}$ is continuous and geometrically symmetric with respect to $\sqrt{a b}$, then the following inequalities for fractional integrals holds:

$$
\begin{aligned}
\left|\begin{array}{c}
\left(\frac{f(a)+f(b)}{2}\right)\left[J_{a+}^{\alpha} g(b)+J_{b-}^{\alpha} g(a)\right] \\
-\left[J_{a+}^{\alpha}(f g)(b)+J_{b-}^{\alpha}(f g)(a)\right]
\end{array}\right| \leq & \frac{\|g\|_{\infty} \ln \alpha+1\left(\frac{b}{a}\right)}{\Gamma(\alpha+1)} \\
& \times\left[\left(1-\frac{1}{2^{\alpha}}\right)\left(\frac{2}{\alpha+1}\right)\right]^{1-\frac{1}{q}}\left[C_{3}(\alpha)\left|f^{\prime}(a)\right|^{q}+C_{4}(\alpha)\left|f^{\prime}(b)\right|^{q}\right]^{\frac{1}{q}},
\end{aligned}
$$

where

$$
\begin{gathered}
C_{3}(\alpha)=\left[\begin{array}{c}
\int_{0}^{\frac{1}{2}}\left[(1-u)^{\alpha}-u^{\alpha}\right](1-u)^{s}\left(a^{1-u} b^{u}\right)^{q} d u \\
+\int_{\frac{1}{2}}^{1}\left[u^{\alpha}-(1-u)^{\alpha}\right](1-u)^{s}\left(a^{1-u} b^{u}\right)^{q} d u
\end{array}\right], \\
C_{4}(\alpha)=\left[\begin{array}{c}
\int_{0}^{\frac{1}{2}}\left[(1-u)^{\alpha}-u^{\alpha}\right] u^{s}\left(a^{1-u} b^{u}\right)^{q} d u \\
+\int_{\frac{1}{2}}^{1}\left[u^{\alpha}-(1-u)^{\alpha}\right] u^{s}\left(a^{1-u} b^{u}\right)^{q} d u
\end{array}\right],
\end{gathered}
$$

with $\alpha>0$.

Proof. Similarly the proof of Theorem 5, using Lemma 1, (8), (9) and power mean inequality we have

$$
\begin{aligned}
\left|\begin{array}{c}
\left(\frac{f(a)+f(b)}{2}\right)\left[J_{a+g}^{\alpha} g(b)+J_{b-}^{\alpha} g(a)\right] \mid \\
-\left[J_{a+}^{\alpha}(f g)(b)+J_{b-}^{\alpha}(f g)(a)\right]
\end{array}\right| & \frac{1}{\Gamma(\alpha)} \int_{0}^{1}\left|\int_{a^{1-u} b^{u}}^{a^{u} b^{1-u}}\left(\ln \frac{b}{s}\right)^{\alpha-1} g(s) \frac{d s}{s}\right|\left|f^{\prime}\left(a^{1-u} b^{u}\right)\right| a^{1-u} b^{u} \ln \left(\frac{b}{a}\right) d u \\
\leq & \frac{\ln \left(\frac{b}{a}\right)}{\Gamma(\alpha)}\left[\int_{0}^{1}\left|\int_{a^{1-u} b^{u}}^{a^{u} b^{1-u}}\left(\ln \frac{b}{s}\right)^{\alpha-1} g(s) \frac{d s}{s}\right| d u\right]^{1-\frac{1}{q}} \\
& \times\left[\int_{0}^{1}\left|\int_{a^{1-u} b^{u}}^{a^{u} b^{1-u}}\left(\ln \frac{b}{s}\right)^{\alpha-1} g(s) \frac{d s}{s}\right|\left|f^{\prime}\left(a^{1-u} b^{u}\right)\right|^{q}\left(a^{1-u} b^{u}\right)^{q} d u\right]^{\frac{1}{q}}
\end{aligned}
$$


Using GA-s convexity of $\left|f^{\prime}\right|^{q}$

$$
\begin{aligned}
& \left|\begin{array}{c}
\left(\frac{f(a)+f(b)}{2}\right)\left[J_{a+}^{\alpha} g(b)+J_{b-}^{\alpha} g(a)\right] \\
-\left[J_{a+}^{\alpha}(f g)(b)+J_{b-}^{\alpha}(f g)(a)\right]
\end{array}\right| \leq \frac{\|g\|_{\infty}^{1-\frac{1}{q}} \ln ^{\alpha\left(1-\frac{1}{q}\right)+1}\left(\frac{b}{a}\right)}{\alpha^{1-\frac{1}{q}} \Gamma(\alpha)}\left[\int_{0}^{\frac{1}{2}}\left[(1-u)^{\alpha}-u^{\alpha}\right] d u+\int_{\frac{1}{2}}^{1}\left[u^{\alpha}-(1-u)^{\alpha}\right] d u\right]^{1-\frac{1}{q}} \\
& \times\left[\int_{0}^{1}\left|\int_{a^{1-u} b^{u}}^{a^{u} b^{1-u}}\left(\ln \frac{b}{s}\right)^{\alpha-1} \frac{d s}{s}\right|\left[(1-u)^{s}\left|f^{\prime}(a)\right|^{q}+u^{s}\left|f^{\prime}(b)\right|^{q}\right]\left(a^{1-u} b^{u}\right)^{q} d u\right]^{\frac{1}{q}} \\
& \leq \frac{\|g\|_{\infty} \ln ^{\alpha+1}\left(\frac{b}{a}\right)}{\Gamma(\alpha+1)}\left[\left(1-\frac{1}{2^{\alpha}}\right)\left(\frac{2}{\alpha+1}\right)\right]^{1-\frac{1}{q}} \\
& \times\left[\begin{array}{c}
\int_{0}^{\frac{1}{2}}\left[(1-u)^{\alpha}-u^{\alpha}\right]\left[(1-u)^{s}\left|f^{\prime}(a)\right|^{q}+u^{s}\left|f^{\prime}(b)\right|^{q}\right]\left(a^{1-u} b^{u}\right)^{q} d u \\
+\int_{\frac{1}{2}}^{1}\left[u^{\alpha}-(1-u)^{\alpha}\right]\left[(1-u)^{s}\left|f^{\prime}(a)\right|^{q}+u^{s}\left|f^{\prime}(b)\right|^{q}\right]\left(a^{1-u} b^{u}\right)^{q} d u
\end{array}\right]^{\frac{1}{q}} \\
& =\frac{\|g\|_{\infty} \ln ^{\alpha+1}\left(\frac{b}{a}\right)}{\Gamma(\alpha+1)}\left[\left(1-\frac{1}{2^{\alpha}}\right)\left(\frac{2}{\alpha+1}\right)\right]^{1-\frac{1}{q}} \\
& \times\left\{\left[\begin{array}{c}
\int_{0}^{\frac{1}{2}}\left[(1-u)^{\alpha}-u^{\alpha}\right](1-u)^{s}\left(a^{1-u} b^{u}\right)^{q} d u \\
+\int_{\frac{1}{2}}^{1}\left[u^{\alpha}-(1-u)^{\alpha}\right](1-u)^{s}\left(a^{1-u} b^{u}\right)^{q} d u
\end{array}\right]\left|f^{\prime}(a)\right|^{q}\right. \\
& \left.+\left[\begin{array}{c}
\int_{0}^{\frac{1}{2}}\left[(1-u)^{\alpha}-u^{\alpha}\right] u^{s}\left(a^{1-u} b^{u}\right)^{q} d u \\
+\int_{\frac{1}{2}}^{1}\left[u^{\alpha}-(1-u)^{\alpha}\right] u^{s}\left(a^{1-u} b^{u}\right)^{q} d u
\end{array}\right]\left|f^{\prime}(b)\right|^{q}\right\}^{\frac{1}{q}}
\end{aligned}
$$

This completes the proof.

\section{Corollary 2. In Theorem 6;}

(1) If we take $\alpha=1$ we have the following Hermite-Hadamard-Fejer inequality for GA-s convex functions which is related to the right-hand side of (3):

$$
\left|\frac{f(a)+f(b)}{2} \int_{a}^{b} \frac{g(x)}{x} d x-\int_{a}^{b} \frac{f(x) g(x)}{x} d x\right| \leq\|g\|_{\infty} \ln ^{2}\left(\frac{b}{a}\right)\left(\frac{1}{2}\right)^{2-\frac{1}{q}}\left[C_{3}(1)\left|f^{\prime}(a)\right|^{q}+C_{4}(1)\left|f^{\prime}(b)\right|^{q}\right]^{\frac{1}{q}}
$$

(2) If we take $g(x)=1$ we have the following Hermite-Hadamard inequality for GA-s convex functions in fractional integral forms which is related to the right-hand side of (4):

$$
\left|\frac{f(a)+f(b)}{2}-\frac{\Gamma(\alpha+1)}{2\left(\ln \frac{b}{a}\right)^{\alpha}}\left[J_{a+}^{\alpha} f(b)+J_{b-}^{\alpha} f(a)\right]\right| \leq \frac{\ln \left(\frac{b}{a}\right)}{2}\left[\left(1-\frac{1}{2^{\alpha}}\right)\left(\frac{2}{\alpha+1}\right)\right]^{1-\frac{1}{q}}\left[C_{3}(\alpha)\left|f^{\prime}(a)\right|^{q}+C_{4}(\alpha)\left|f^{\prime}(b)\right|^{q}\right]^{\frac{1}{q}},
$$

(3) If we take $\alpha=1$ and $g(x)=1$ we have the following Hermite-Hadamard inequality for GA-s convex functions:

$$
\left|\frac{f(a)+f(b)}{2}-\frac{1}{\ln \frac{b}{a}} \int_{a}^{b} \frac{f(x)}{x} d x\right| \leq \ln \left(\frac{b}{a}\right)\left(\frac{1}{2}\right)^{2-\frac{1}{q}}\left[C_{3}(1)\left|f^{\prime}(a)\right|^{q}+C_{4}(1)\left|f^{\prime}(b)\right|^{q}\right]^{\frac{1}{q}} .
$$

Theorem 7. Let $f: I \subset(0, \infty) \rightarrow \mathbb{R}$ be a differentiable mapping on $I^{\circ}$ where $a, b \in I$ with $a<b$ and $f^{\prime} \in L[a, b]$. If $\left|f^{\prime}\right|^{q}$, $q>1$ is GA-s convex on $[a, b], g:[a, b] \rightarrow \mathbb{R}$ is continuous and geometrically symmetric with respect to $\sqrt{a b}$, then the following inequalities for fractional integrals holds: 
(i)

$$
\left|\begin{array}{c}
\left(\frac{f(a)+f(b)}{2}\right)\left[J_{a+g}^{\alpha} g(b)+J_{b-}^{\alpha} g(a)\right] \\
-\left[J_{a+}^{\alpha}(f g)(b)+J_{b-}^{\alpha}(f g)(a)\right]
\end{array}\right| \leq \frac{\|g\|_{\infty} \ln ^{\alpha+1}\left(\frac{b}{a}\right)}{\Gamma(\alpha+1)}\left[\frac{2}{\alpha p+1}\left(1-\frac{1}{2^{\alpha p}}\right)\right]^{\frac{1}{p}}\left[C_{5}\left|f^{\prime}(a)\right|^{q}+C_{6}\left|f^{\prime}(b)\right|^{q}\right]^{\frac{1}{q}}
$$

with $\alpha>0$.

(ii)

$$
\left|\begin{array}{c}
\left(\frac{f(a)+f(b)}{2}\right)\left[J_{a+}^{\alpha} g(b)+J_{b-}^{\alpha} g(a)\right] \\
-\left[J_{a+}^{\alpha}(f g)(b)+J_{b-}^{\alpha}(f g)(a)\right]
\end{array}\right| \leq \frac{\|g\|_{\infty} \ln ^{\alpha+1}\left(\frac{b}{a}\right)}{\Gamma(\alpha+1)}\left[\frac{1}{\alpha p+1}\right]^{\frac{1}{p}}\left[C_{5}\left|f^{\prime}(a)\right|^{q}+C_{6}\left|f^{\prime}(b)\right|^{q}\right]^{\frac{1}{q}}
$$

for $0<\alpha \leq 1$. Where

$$
C_{5}=\int_{0}^{1}(1-u)^{s}\left(a^{1-u} b^{u}\right)^{q} d u, C_{6}=\int_{0}^{1} u^{s}\left(a^{1-u} b^{u}\right)^{q} d u
$$

and $1 / p+1 / q=1$

Proof. (i) Using Lemma 1, (8), (9), Hölder's inequality and GA-s convexity of $\left|f^{\prime}\right|^{q}$ we have

$$
\begin{aligned}
& \left|\begin{array}{c}
\left(\frac{f(a)+f(b)}{2}\right)\left[J_{a+}^{\alpha} g(b)+J_{b-}^{\alpha} g(a)\right] \\
-\left[J_{a+}^{\alpha}(f g)(b)+J_{b-}^{\alpha}(f g)(a)\right]
\end{array}\right| \leq \frac{1}{\Gamma(\alpha)} \int_{0}^{1}\left|\int_{a^{1-u} b^{u}}^{a^{u} b^{1-u}}\left(\ln \frac{b}{s}\right)^{\alpha-1} g(s) \frac{d s}{s}\right|\left|f^{\prime}\left(a^{1-u} b^{u}\right)\right| a^{1-u} b^{u} \ln \left(\frac{b}{a}\right) d u \\
& \leq \frac{\ln \left(\frac{b}{a}\right)}{\Gamma(\alpha)}\left[\int_{0}^{1}\left|\int_{a^{1-u} b^{u}}^{a^{u} b^{1-u}}\left(\ln \frac{b}{s}\right)^{\alpha-1} g(s) \frac{d s}{s}\right|^{p} d u\right]^{\frac{1}{p}}\left[\int_{0}^{1}\left|f^{\prime}\left(a^{1-u} b^{u}\right)\right|^{q}\left(a^{1-u} b^{u}\right)^{q} d u\right]^{\frac{1}{q}} \\
& \leq \frac{\|g\|_{\infty} \ln ^{\alpha+1}\left(\frac{b}{a}\right)}{\Gamma(\alpha+1)}\left[\int_{0}^{\frac{1}{2}}\left[(1-u)^{\alpha}-u^{\alpha}\right]^{p} d u+\int_{\frac{1}{2}}^{1}\left[u^{\alpha}-(1-u)^{\alpha}\right]^{p} d u\right]^{\frac{1}{p}} \\
& \times\left[\int_{0}^{1}\left[(1-u)^{s}\left|f^{\prime}(a)\right|^{q}+u^{s}\left|f^{\prime}(b)\right|^{q}\right]\left(a^{1-u} b^{u}\right)^{q} d u\right]^{\frac{1}{q}} \\
& =\frac{\|g\|_{\infty} \ln ^{\alpha+1}\left(\frac{b}{a}\right)}{\Gamma(\alpha+1)}\left[\int_{0}^{\frac{1}{2}}\left[(1-u)^{\alpha}-u^{\alpha}\right]^{p} d u+\int_{\frac{1}{2}}^{1}\left[u^{\alpha}-(1-u)^{\alpha}\right]^{p} d u\right]^{\frac{1}{p}} \\
& \times\left[\left(\int_{0}^{1}(1-u)^{s}\left(a^{1-u} b^{u}\right)^{q} d u\right)\left|f^{\prime}(a)\right|^{q}+\left(\int_{0}^{1} u^{s}\left(a^{1-u} b^{u}\right)^{q} d u\right)\left|f^{\prime}(b)\right|^{q}\right]^{\frac{1}{q}} \\
& \leq \frac{\|g\|_{\infty} \ln ^{\alpha+1-\frac{1}{q}}\left(\frac{b}{a}\right)}{q^{\frac{1}{q}} \Gamma(\alpha+1)}\left[\int_{0}^{\frac{1}{2}}(1-u)^{\alpha p}-u^{\alpha p} d u+\int_{\frac{1}{2}}^{1} u^{\alpha p}-(1-u)^{\alpha p} d u\right]^{\frac{1}{p}} \\
& \times\left[\left(\int_{0}^{1}(1-u)^{s}\left(a^{1-u} b^{u}\right)^{q} d u\right)\left|f^{\prime}(a)\right|^{q}+\left(\int_{0}^{1} u^{s}\left(a^{1-u} b^{u}\right)^{q} d u\right)\left|f^{\prime}(b)\right|^{q}\right]^{\frac{1}{q}} \\
& =\frac{\|g\|_{\infty} \ln ^{\alpha+1}\left(\frac{b}{a}\right)}{\Gamma(\alpha+1)}\left[\frac{2}{\alpha p+1}\left(1-\frac{1}{2^{\alpha p}}\right)\right]^{\frac{1}{p}}\left[C_{5}\left|f^{\prime}(a)\right|^{q}+C_{6}\left|f^{\prime}(b)\right|^{q}\right]^{\frac{1}{q}} \text {. }
\end{aligned}
$$

Here we use

$$
\left[(1-t)^{\alpha}-t^{\alpha}\right]^{p} \leq(1-t)^{\alpha p}-t^{\alpha p}
$$

for $t \in[0,1 / 2]$ and

$$
\left[t^{\alpha}-(1-t)^{\alpha}\right]^{p} \leq t^{\alpha p}-(1-t)^{\alpha p}
$$

for $t \in[1 / 2,1]$, which follows from

$$
(A-B)^{q} \leq A^{q}-B^{q},
$$


for any $A \geq B \geq 0$ and $q \geq 1$. Hence the inequality (11) is proved.

(ii) The inequality (12) is easily proved using (13) and Lemma 2.

\section{Corollary 3. In Theorem 7;}

(i) In (11); (1) If we take $\alpha=1$ we have the following Hermite-Hadamard-Fejer inequality for GA-s convex functions which is related to the right-hand side of $(3)$ :

$$
\left|\frac{f(a)+f(b)}{2} \int_{a}^{b} \frac{g(x)}{x} d x-\int_{a}^{b} \frac{f(x) g(x)}{x} d x\right| \leq \frac{\|g\|_{\infty} \ln ^{2}\left(\frac{b}{a}\right)}{2}\left[\frac{2}{p+1}\left(1-\frac{1}{2^{p}}\right)\right]^{\frac{1}{p}}\left[C_{5}\left|f^{\prime}(a)\right|^{q}+C_{6}\left|f^{\prime}(b)\right|^{q}\right]^{\frac{1}{q}}
$$

(2) If we take $g(x)=1$ we have the following Hermite-Hadamard inequality for GA-s convex functions in fractional integral forms which is related to the right-hand side of (4):

$$
\left|\frac{f(a)+f(b)}{2}-\frac{\Gamma(\alpha+1)}{2\left(\ln \frac{b}{a}\right)^{\alpha}}\left[J_{a+}^{\alpha} f(b)+J_{b-}^{\alpha} f(a)\right]\right| \leq \frac{\ln \left(\frac{b}{a}\right)}{2}\left[\frac{2}{\alpha p+1}\left(1-\frac{1}{2^{\alpha p}}\right)\right]^{\frac{1}{p}}\left[C_{5}\left|f^{\prime}(a)\right|^{q}+C_{6}\left|f^{\prime}(b)\right|^{q}\right]^{\frac{1}{q}}
$$

(3) If we take $\alpha=1$ and $g(x)=1$ we have the following Hermite-Hadamard inequality for GA-s convex functions:

$$
\left|\frac{f(a)+f(b)}{2}-\frac{1}{\ln \frac{b}{a}} \int_{a}^{b} \frac{f(x)}{x} d x\right| \leq \frac{\ln ^{1}\left(\frac{b}{a}\right)}{2}\left[\frac{2}{p+1}\left(1-\frac{1}{2^{p}}\right)\right]^{\frac{1}{p}}\left[C_{5}\left|f^{\prime}(a)\right|^{q}+C_{6}\left|f^{\prime}(b)\right|^{q}\right]^{\frac{1}{q}} .
$$

(ii) In (12);

(1) If we take $\alpha=1$ we have the following Hermite-Hadamard-Fejer inequality for GA-s convex functions which is related to the right-hand side of (3):

$$
\left|\frac{f(a)+f(b)}{2} \int_{a}^{b} \frac{g(x)}{x} d x-\int_{a}^{b} \frac{f(x) g(x)}{x} d x\right| \leq \frac{\|g\|_{\infty} \ln ^{2}\left(\frac{b}{a}\right)}{2}\left[\frac{1}{p+1}\right]^{\frac{1}{p}}\left[C_{5}\left|f^{\prime}(a)\right|^{q}+C_{6}\left|f^{\prime}(b)\right|^{q}\right]^{\frac{1}{q}},
$$

(2) If we take $g(x)=1$ we have the following Hermite-Hadamard inequality for GA-s convex functions in fractional integral forms which is related to the right-hand side of (4):

$$
\left|\frac{f(a)+f(b)}{2}-\frac{\Gamma(\alpha+1)}{2\left(\ln \frac{b}{a}\right)^{\alpha}}\left[J_{a+}^{\alpha} f(b)+J_{b-}^{\alpha} f(a)\right]\right| \leq \frac{\ln \left(\frac{b}{a}\right)}{2}\left[\frac{1}{\alpha p+1}\right]^{\frac{1}{p}}\left[C_{5}\left|f^{\prime}(a)\right|^{q}+C_{6}\left|f^{\prime}(b)\right|^{q}\right]^{\frac{1}{q}}
$$

(3) If we take $\alpha=1$ and $g(x)=1$ we have the following Hermite-Hadamard inequality for GA-s convex functions:

$$
\left|\frac{f(a)+f(b)}{2}-\frac{1}{\ln \frac{b}{a}} \int_{a}^{b} \frac{f(x)}{x} d x\right| \leq \frac{\ln \left(\frac{b}{a}\right)}{2}\left[\frac{1}{p+1}\right]^{\frac{1}{p}}\left[C_{5}\left|f^{\prime}(a)\right|^{q}+C_{6}\left|f^{\prime}(b)\right|^{q}\right]^{\frac{1}{q}} .
$$

\section{Conflict of interests}

The authors declare that there is no conflict of interests regarding the publication of this paper.

\section{References}

[1] L. Fejér, Uber die Fourierreihen, II, Math. Naturwise. Anz Ungar. Akad., Wiss, 24 (1906), 369-390, (in Hungarian). 
[2] J. Hadamard, Étude sur les propriétés des fonctions entières et en particulier d'une fonction considérée par Riemann, J. Math. Pures Appl., 58 (1893), 171-215.

[3] İ. İşcan, Hermite-Hadamard-Fejer type inequalities for convex functions via fractional integrals, arXiv preprint arXiv:1404.7722 (2014).

[4] İ. İşcan, Generalization of different type integral inequalities for $s$-convex functions via fractional integrals, Applicable Analysis, 2013. doi: 10.1080/00036811.2013.851785.

[5] İ. İşcan, New general integral inequalities for quasi-geometrically convex functions via fractional integrals, J. Inequal. Appl., 2013(491) (2013), 15 pages.

[6] İ. İşcan, On generalization of different type integral inequalities for $s$-convex functions via fractional integrals, Mathematical Sciences and Applications E-Notes, 2(1) (2014), 55-67.

[7] İ. İşcan, S. Turhan, S. Maden, Some Hermite-Hadamard-Fejer type inequalities for harmonically convex functions via fractional integral, New Trends in Mathematical Sciences, 4(2) (2016), 1-10.

[8] İ. İşcan, M. Kunt, Hermite-Hadamard-Fejer type inequalities for GA-convex functions via fractional integrals, RGMIA Research Report Collection, 18(2015), Article 101, 13pp.

[9] A. A. Kilbas, H. M. Srivastava, J. J. Trujillo, Theory and applications of fractional differential equations. Elsevier, Amsterdam (2006).

[10] M. A. Latif, S. S. Dragomir and E. Momaniat, Some Fejer type integral inequalities for geometrically-arithmetically-convex functions with applications, RGMIA Research Report Collection, 18(2015), Article 15, 18pp.

[11] C. P. Niculescu, Convexity according to the geometric mean, Math. Inequal. Appl. 3 (2) (2000), 155-167. Available online at http://dx.doi.org/10.7153/mia-03-19.

[12] C. P. Niculescu, Convexity according to means, Math. Inequal. Appl. 6 (4) (2003), 571-579. Available online at http://dx.doi.org/10.7153/mia-06-53.

[13] A.P. Prudnikov, Y.A. Brychkov and O.I. Marichev, Integral and series. In: Elementary Functions, vol. 1. Nauka, Moscow, 1981.

[14] M.Z. Sarıkaya, On new Hermite Hadamard Fejér type integral inequalities, Stud. Univ. Babeş-Bolyai Math. 57(3) (2012), 377-386.

[15] Erhan Set, İ. İşcan, M. Zeki Sarikaya, M. Emin Ozdemir, On new inequalities of Hermite-Hadamard-Fejer type for convex functions via fractional integrals, Applied Mathematics and Computation, 259 (2015) 875-881.

[16] Y. Shuang, H. P. Yin, F. Qi, Hermite-Hadamard type integral inequalities for geometrically-arithmetically s-convex functions, Analysis (Munich) 33 (2) (2013) 197-2008.

[17] K.-L. Tseng, G.-S. Yang and K.-C. Hsu, Some inequalities for differentiable mappings and applications to Fejér inequality and weighted trapezoidal formula, Taiwanese journal of Mathematics, 15(4) (2011), 1737-1747.

[18] J. Wang, X. Li, M. Fečkan and Y. Zhou, Hermite-Hadamard-type inequalities for Riemann-Liouville fractional integrals via two kinds of convexity, Appl. Anal., 92(11) (2012), 2241-2253. doi:10.1080/00036811.2012.727986

[19] J. Wang, C. Zhu and Y. Zhou, New generalized Hermite-Hadamard type inequalities and applications to special means, J. Inequal. Appl., 2013(325) (2013), 15 pages. 